\title{
Basic Diagnostic Work-Up Is More Complete in Rural than in Urban Areas for Patients with Dementia: Results of a Swedish Dementia Registry Study
}

\author{
Mandy Roheger ${ }^{\mathrm{a}}$, Maria Eriksdotter ${ }^{\mathrm{b}, \mathrm{c}}$, Karin Westling ${ }^{\mathrm{c}}$, Elke Kalbe ${ }^{\mathrm{a}}$ and Sara Garcia-Ptacek ${ }^{\mathrm{b}, \mathrm{d}, *}$ \\ ${ }^{\mathrm{a}}$ Medical Faculty and University Hospital Cologne, Cologne, Germany \\ ${ }^{\mathrm{b}}$ Division of Clinical Geriatrics, Department of Neurobiology, Care Sciences and Society, \\ Karolinska Institutet, Stockholm, Sweden \\ ${ }^{\mathrm{c}}$ Theme Aging, Karolinska University Hospital, Stockholm, Sweden \\ ${ }^{\mathrm{d}}$ Neurology Section, Department of Internal Medicine, Södersjukhuset, Stockholm, Sweden
}

\begin{abstract}
.
Background: Life in rural areas differs from life in urban areas not only in geographical conditions, but also in health care structure.

Objective: Our aim is to compare the diagnostic process and the management of dementia in rural and urban areas of Sweden. Methods: We performed a cross-sectional study of patients with dementia living in rural $(n=16,428)$, intermediate $(n=18,033)$, and urban $(n=23,680)$ areas in Sweden including patients registered from 2007 through 2014 in the Swedish Dementia Registry (SveDem). Descriptive statistics are shown. Odds ratios with 95\% CI are presented for basic diagnostic examinations in rural compared to intermediate and urban areas, adjusted for age, sex, type of care (primary versus specialist), and comorbidities. Analyses were also stratified for diagnostic care unit (primary versus specialist).

Results: Patients who lived in rural areas were more likely to receive a complete basic examination, MMSE examination, Clock test, blood analysis, and neuro-imaging, compared to patients living in urban areas, and also compared to patients living in intermediate areas. Sex differences were seen in nearly all domains, with men receiving more diagnostic work-up than women. Stratified analyses show that in primary care, the complete basic examination is less frequently performed in urban and intermediate areas compared to rural areas.

Conclusion: There are differences in diagnostic work-up for dementia between rural, intermediate, and urban areas in Sweden. These results should be considered in future healthcare decisions to ensure equality of health care across rural and urban areas.
\end{abstract}

Keywords: Dementia, diagnostic work-up, epidemiology, living typology, SveDem

\footnotetext{
${ }^{*}$ Correspondence to: Sara Garcia-Ptacek, Department of Neurobiology, Care Sciences and Society (NVS), Division of Clinical Geriatrics, Neo, Blickagången 16, 14152 Huddinge, Sweden. E-mail: sara.garcia-ptacek@ki.se.
}

\section{INTRODUCTION}

Geographical variations (such as living in a rural, intermediate, or urban area) in dementia prevalence and incidence may indicate important socio-environmental contributions to dementia etiol- 
ogy [1] and are therefore important to investigate. A systematic review and meta-analysis by Russ et al. (2002) including 51 articles on geographical variation in the prevalence and incidence of dementia showed that rural living and early life rural living may be associated with increased rates of Alzheimer's disease (AD) due to a higher probability to be exposed to potentially modifiable risk factors [1]. Life in rural areas differs from life in urban areas not only in geographical conditions, but also in population age structures, education, income, access to public transport, nursing home placement, and access to specialist treatment and overall health care structures [2-5]. This leads to the question of the processes and quality of diagnostic work-up for patients with dementia and possible inequalities regarding diagnostic work-up processes between patients with dementia living in rural, intermediate, and urban areas in Sweden.

The Swedish National Board of Health and Welfare published guidelines in 2010 in which they defined the contents of a basic dementia work-up [6], which should include a short cognitive screening using the Mini Mental State Examination (MMSE) [7] and the Clock Test [8], a blood analysis (calcium, TSH, and either homocysteine or B12 and folate), and neuroimaging (computed tomography $[\mathrm{CT}]$ or magnetic resonance imaging [MRI] of the brain). Additionally, a structured interview with the patient and a reliable reporter and a physical exam are required, including a functional assessment. For the present study, we used the Swedish Dementia Registry (SveDem), which is a national quality registry launched in 2007 [9]. Part of the purpose of SveDem is to evaluate the quality indicators established by the Swedish Board of Health and Welfare in 2010 [9], to establish it as a tool for instituting and evaluating public health policy to improve dementia care. Therefore, and due to the fact that there is still a lack of research concerning diagnostic work-up processes and outcomes for patients with dementia in different living settings, the aim of the present study was to describe and compare characteristics and diagnostic work-up procedures for patients with dementia living in rural, intermediate and urban areas in Sweden. We expected a greater coverage of diagnostic work-up procedures in urban compared to intermediate and rural areas. Furthermore, we wanted to examine the associations between living areas and different diagnostic workup processes. We expected that patients living in rural areas were less likely to receive the different diagnostic work-up processes, when adjusting for age, sex,
Charlson Comorbidity Index, type of care, and total dependency ratio.

\section{MATERIALS AND METHODS}

We analyzed cross-sectional data of patients with dementia living in rural, intermediate, and urban areas in Sweden including patients registered in SveDem, the Swedish nationwide registry of dementia disorders [9]. SveDem has the aim to register patients with dementia at the time of the dementia diagnosis and monitor their care, as already described in detail [9, 10]. Briefly, patients are registered by a specialist or primary care unit. In Sweden, the basic health and medical care is generally referred to as primary care. The primary care services are comprised of general medical practitioners offering medical examinations, care and treatment of most common conditions and illnesses. Specialist care, however, is defined as care that requires more specialized medical measures than what is available through primary care. There are around 1170 primary care units and 58 specialist units in Sweden in 2018 (SveDem yearly report, 2018). The registered variables include information on patients' demographics, living situation, dementia type, and diagnostic work-up including blood analyses, cognitive evaluation using MMSE [7], Clock test, as well as either CT or MRI as imaging methods. Furthermore, this information was completed with data on medication use obtained from the Swedish Prescribed Drug Register (PDR, which has a coverage close to $100 \%$ ) [11], and data on comorbidities which were obtained from the National Inpatient Registry (NIR), covering all specialist clinic and hospital diagnoses [12].

\section{Study population}

The study population included patients with dementia registered from 2007 through 2014. Of 58,154 patients in SveDem, $n=13$ were excluded due to missing values about information on living typology (rural, intermediate, urban). Hence, 58,141 patients were included.

\section{Variable definitions}

The rural, intermediate, and urban typology for the Swedish geographical regions were defined for all 290 Swedish municipalities (kommuner) according to the Classification of Swedish municipalities 2017 produced by the Swedish Association of Local Authorities and Regions [13]. The classification 
consists of three main groups (A: large cities and municipalities near large cities; B: medium-sized towns and municipalities near medium-sized towns; C: smaller towns/urban areas and rural municipalities) in which the municipalities are further divided into a total of nine groups based on structural parameters such as population and commuting patterns. For a more detailed overview, see the map in the Supplementary Material.

From SveDem, we used information on dementia diagnosis, dementia type, type of care (specialist versus primary care), basic examination at time of diagnosis, i.e., blood analysis, cognitive evaluation with the MMSE, the clock test, as well as imaging procedures (CT and/or MRI). The variable "Basic examination" was calculated and considered as complete if the patient had received MMSE, clock test, blood tests, and neuroimaging (CT and/or MRI).

The total dependency ratio was defined as the proportion of people aged 20-64 working in a specific region compared to older and younger people. The variable was calculated using the average of the years 2007 until 2014 [14].

Furthermore, the Charlson Comorbidity Index (CCI) [15] was calculated using ICD-10 coded diagnoses from the NIR and integrated in the analyses.

\section{Statistical analysis}

The IBM Statistical Package for Social Sciences [16] for Windows, version 22, was used for the analyses.

For the descriptive statistics, continuous variables were summarized as means and standard deviations or as medians and interquartile ranges, as appropriate. For categorical variables, data were presented as number of cases and percentages. To calculate p-values for differences, ANOVAs were used for continuous, and chi-square tests for categorical variables. Values were presented in total and for sex differences.

Binary logistic regressions were applied to estimate odds ratios (ORs) with 95\% confidence intervals (CIs) for associations of basic examination procedures and rural versus intermediate versus urban residency at the time of dementia diagnosis. The fully adjusted model was controlled for age, sex, CCI, total dependency ratio, and type of care (specialist versus primary).

Furthermore, we conducted binary logistic regressions stratified for type of care (primary versus specialist) with $95 \%$ CIs for associations of basic examination procedures and rural versus intermediate versus urban residency at the time of dementia diagnosis. Models were adjusted for age, sex, CCI, and total dependency ratio.

\section{RESULTS}

\section{Characteristics of study population}

Of the 58,141 patients with dementia included, $n=16,428 \quad(28.26 \%)$ lived in rural, $n=18,033$ $(31.02 \%)$ in intermediate, and $n=23,680(40.72 \%)$ lived in urban areas in Sweden. Demographic characteristics of the patients by sex and living typology are presented in Table 1 . Many patients were living alone (rural: $46.6 \%$, intermediate: $42.7 \%$, urban: $50.8 \%$ ), even though more people in urban areas compared to intermediate and rural ones were living alone $(p<0.001)$. AD and mixed dementia were the most common dementia types (AD: rural: $32.1 \%$; intermediate: $32.8 \%$; urban: $27.6 \%$; Mixed: rural: $12.3 \%$; intermediate: $11.7 \%$; urban: $27.4 \%$ ). The majority of patients were in the mild stage of dementia as defined by MMSE at the time of dementia diagnosis (rural: $M=20.97 ; S D=4.88$; intermediate: $M=20.8$, $S D=5.02$; urban: $M=20.8, S D=5.18)$. Overall, the proportion of patients who received a full basic examination in all three areas ranged from 65 to $75 \%$ (rural: $71.1 \%$; intermediate: $65.8 \%$; urban: $74.1 \%$ ). Gender differences could be observed in nearly all domains of diagnostic work-up (except for MRI, and EEG in rural and intermediate areas), indicating that men received more diagnostic work-up than women. The only exception was the performance of the MMSE in rural areas (men: 93.7\%; women: 94.0\%).

\section{Results of the logistic regressions}

In adjusted analyses, patients living in urban areas were less likely to receive the complete basic examination $(0.81,95 \%$ CI: $0.76-0.86)$, i.e., MMSE examination $(0.58,95 \% \mathrm{CI}: 0.51-0.67)$, Clock test (0.78, 95\% CI: $0.72-0.84)$, blood analysis $(0.75,95 \%$ CI: $0.66-0.86)$, and neuro-imaging $(0.91,95 \% \mathrm{CI}$ : 0.83-0.99) compared to rural, and also compared to intermediate areas (BE: 0.66, 95\% CI: 0.63-0.70; MMSE: 0.54, 95\% CI: 0.48-0.60; Clock: 0.76, 95\% CI: 0.71-0.82; blood: 0.78 , 95\% CI: $0.70-0.87$; imaging: $0.73,95 \%$ CI: $0.68-0.76)$. The models are adjusted for age, sex, CCI, type of care (specialist versus primary), and total dependency ratio and are presented in Table 2. 


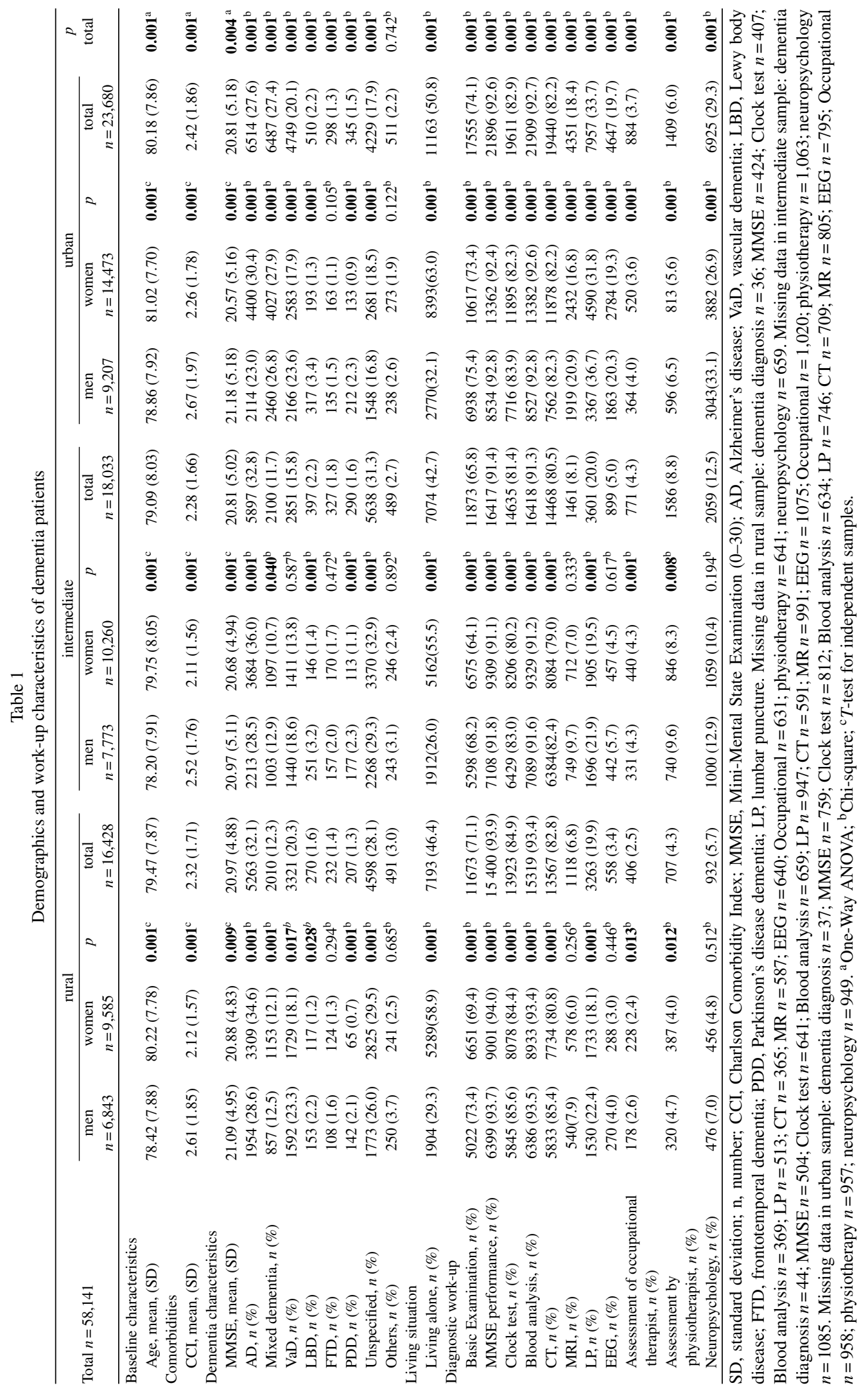


Table 2

Overall logistic regressions of basic examination, MMSE scores, blood analysis, and CT or MRI at dementia diagnosis

\begin{tabular}{|c|c|c|c|c|c|}
\hline Adjusted Analyses & $\begin{array}{c}\text { Analysis 1: } \\
\text { Basic Examination }\end{array}$ & $\begin{array}{l}\text { Analysis 2: } \\
\text { MMSE scores }\end{array}$ & $\begin{array}{l}\text { Analysis 3: } \\
\text { Clock Test }\end{array}$ & $\begin{array}{c}\text { Analysis 4: } \\
\text { Blood Analysis }\end{array}$ & $\begin{array}{c}\text { Analysis 5: } \\
\text { CT and/or MRI }\end{array}$ \\
\hline \multicolumn{6}{|l|}{ Living situation } \\
\hline \multicolumn{6}{|l|}{ Rural } \\
\hline Urban & $0.81(0.76-0.86)$ & $0.58(0.51-0.67)$ & $0.78(0.72-0.84)$ & $0.75(0.66-0.86)$ & $0.91(0.83-0.99)$ \\
\hline Intermediate & $0.66(0.63-0.70)$ & $0.54(0.48-0.60)$ & $0.76(0.71-0.82)$ & $0.78(0.70-0.87)$ & $0.73(0.68-0.76)$ \\
\hline \multicolumn{6}{|l|}{ Age } \\
\hline$<81$ years old & $1.01(1.00-1.01)$ & $1.05(1.05-1.06)$ & $1.01(1.01-1.02)$ & $1.02(1.01-1.03)$ & $0.99(0.99-1.00)$ \\
\hline$\geq 81$ years old & $0.91(0.90-0.91)$ & $0.91(0.91-0.93)$ & $0.92(0.92-0.93)$ & $0.94(0.93-0.95)$ & $0.87(0.88-0.89)$ \\
\hline Sex & $1.06(1.02-1.11)$ & $0.96(0.88-1.05)$ & $1.06(1.01-1.12)$ & $0.96(0.88-1.05)$ & $1.11(1.05-1.18)$ \\
\hline Charlson Comorbidity Index & $0.98(0.97-0.99)$ & $0.97(0.95-0.99)$ & $0.97(0.96-0.99)$ & $0.96(0.94-0.98)$ & $1.01(1.00-1.03)$ \\
\hline Type of Care & $0.36(0.35-0.38)$ & $0.30(0.27-0.33)$ & $0.58(0.55-0.62)$ & $0.58(0.53-0.64)$ & $0.18(0.17-0.20)$ \\
\hline Total dependency ratio & $0.99(0.99-0.99)$ & $1.00(0.99-1.00)$ & $1.00(0.99-1.00)$ & $0.99(0.99-1.00)$ & $0.98(0.98-0.99)$ \\
\hline Constant & 8.63 & 2.52 & 6.66 & 19.52 & 178.63 \\
\hline
\end{tabular}

Results obtained from binary logistic regressions for the association between Basic Examination, MMSE sores, Clock Test, Blood Analysis, CT and/or MRT, and living typology (urban versus rural). Results are adjusted for age, sex, Charlson Comorbidity Index, total dependency ratio and type of care (primary versus specialist). In all analyses, rural living typology is the reference category, as well as female persons and specialized care. Missings in adjusted analyses: analysis 1: $n=6,942$; analysis $2: n=8,525$; analysis 3:8,671; analysis 4: $n=8,506$; analysis 5: $n=6,942$. Bold scores indicate a $p$-value $<0.05$.

In stratified analyses by level of care, patients living in urban areas were less likely in primary care to receive the basic examination $(0.63,95 \%$ CI: 0.58-0.69), MMSE examination $(0.46,95 \% \mathrm{CI}$ : 0.39-0.55), Clock test (0.63, 95\% CI: $0.57-0.70)$, blood analysis (0.58, 95\% CI: 0.49-0.68), and neuroimaging $(0.84,95 \% \mathrm{CI}: 0.76-0.93)$ compared to rural, and also compared to intermediate areas (BE: 0.60, 95\% CI: 0.56-0.64; MMSE: 0.53 , 95\% CI: 0.47-0.61; Clock: 0.66, 95\% CI: 0.61-0.72; blood: 0.76, 95\% CI: 0.67-0.87; imaging: $0.71,95 \% \mathrm{CI}$ : $0.66-0.76$ ) in primary care. In specialist care, however, patients living in urban areas don't significantly receive basic examination (1.05, 95\% CI: 0.94-1.16), Clock tests (1.07, 95\% CI: 0.94-1.22), and imaging (1.04, 95\% CI: 0.86-1.27), whereas patients living in intermediate areas receive less basic examination (0.83, 95\% CI: 0.75-0.92), MMSE testing (0.52, 95\% CI: $0.41-0.67)$, blood analysis $(0.80,95 \% \mathrm{CI}$ : $0.65-0.99)$, and imaging (0.78, 95\% CI: 0.64-0.94). The stratified models are presented in Table 3.

\section{DISCUSSION}

As life in rural areas differs from life in intermediate and urban areas in socio-demographic and health-related variables [4], it is important to evaluate the quantity of diagnostic work-up processes and to ensure that patients with dementia in all areas in Sweden receive a complete basic examination. National diagnostic guidelines are one step in this direction [6]. Yet, the present study is, to the authors' best knowledge, the first study that examines geographical differences in diagnostic work-up processes for patients with dementia living in Sweden.

The present study demonstrates some differences between diagnostic work-up processes in rural, intermediate, and urban living typologies. The overall basic examination rate in all three living typologies can be improved, showing that around $25-35 \%$ of the patients do not receive a full basic examination. This result was also supported by Garcia-Ptacek et al. (2017), who investigated the AD subgroup. They showed that one or more tests of the basic examination were missing in $47 \%$ of patients with $\mathrm{AD}$ [17]. Therefore, an improvement of the overall quality of basic examination is needed. However, encouragingly though, the latest SveDem yearly report shows an increase in the proportion of basic examinations in primary care from $46 \%$ in 2011 to $70 \%$ in 2017 [18].

Surprisingly, patients living in rural areas were more likely to receive the basic examination, MMSE, Clock test, blood analysis, and neuroimaging, compared to patients living in urban, and also compared to patients living in intermediate areas in adjusted analyses. Sweden is a large and sparsely populated country; therefore, one could assume that in some cases, sending a patient hours away for, e.g., a CT scan may not be common practice. Yet, our study shows the opposite results. We can only speculate on the possible reasons; however, this result could also be explained by a sampling bias generated by the coverage of around $40 \%$ of the SveDem data. It could be possible that patients living in rural areas are more likely to get registered in SveDem when they also received the full basic examination. 
Table 3

Stratified logistic regressions for type of care (primary versus specialist) of basic examination, MMSE scores, Blood analysis, and CT or MRI at dementia diagnosis

\begin{tabular}{|c|c|c|c|c|c|}
\hline $\begin{array}{l}\text { Stratified analyses } \\
\text { Primary care }\end{array}$ & $\begin{array}{c}\text { Analysis 1: } \\
\text { Basic Examination }\end{array}$ & $\begin{array}{l}\text { Analysis 2: } \\
\text { MMSE scores }\end{array}$ & $\begin{array}{l}\text { Analysis 3: } \\
\text { Clock Test }\end{array}$ & $\begin{array}{c}\text { Analysis 4: } \\
\text { Blood Analysis }\end{array}$ & $\begin{array}{c}\text { Analysis 5: } \\
\text { CT and/or MRI }\end{array}$ \\
\hline \multicolumn{6}{|l|}{ Living situation } \\
\hline \multicolumn{6}{|l|}{ Rural } \\
\hline Urban & $0.63(0.58-0.69)$ & $0.46(0.39-0.55)$ & $0.63(0.57-0.70)$ & $0.58(0.49-0.68)$ & $0.84(0.76-0.93)$ \\
\hline Intermediate & $0.60(0.56-0.64)$ & $0.53(0.47-0.61)$ & $0.66(0.61-0.72)$ & $0.76(0.67-0.87)$ & $0.71(0.66-0.76)$ \\
\hline \multicolumn{6}{|l|}{ Age } \\
\hline$<81$ years old & $1.01(1.01-1.02)$ & $1.07(1.05-1.08)$ & $1.02(1.01-1.03)$ & $1.01(1.00-1.03)$ & $0.99(0.99-1.00)$ \\
\hline$\geq 81$ years old & $0.91(0.90-0.91)$ & $0.92(0.90-0.93)$ & $0.93(0.93-0.94)$ & $0.93(0.92-0.94)$ & $0.88(0.87-0.89)$ \\
\hline Sex & $1.09(1.04-1.15)$ & $1.05(0.95-1.16)$ & $1.08(1.02-1.16)$ & $1.02(0.92-1.14)$ & $1.12(1.06-1.20)$ \\
\hline Charlson Comorbidity Index & $1.00(0.98-1.01)$ & $0.97(0.95-1.00)$ & $1.00(0.98-1.01)$ & $0.95(0.93-0.98)$ & $1.01(1.00-1.02)$ \\
\hline Total dependency ratio & $0.97(0.97-0.98)$ & $0.99(0.98-1.00)$ & $0.99(0.98-0.99)$ & $0.99(0.98-0.99)$ & $0.98(0.97-0.98)$ \\
\hline Constant & 7.28 & 0.46 & 3.48 & 33.52 & 61.57 \\
\hline $\begin{array}{l}\text { Stratified analyses } \\
\text { Specialist care }\end{array}$ & $\begin{array}{c}\text { Analysis 1: } \\
\text { Basic Examination }\end{array}$ & $\begin{array}{c}\text { Analysis 2: } \\
\text { MMSE scores }\end{array}$ & $\begin{array}{l}\text { Analysis 3: } \\
\text { Clock Test }\end{array}$ & $\begin{array}{c}\text { Analysis 4: } \\
\text { Blood Analysis }\end{array}$ & $\begin{array}{c}\text { Analysis 5: } \\
\text { CT and/or MRI }\end{array}$ \\
\hline \multicolumn{6}{|l|}{ Living situation } \\
\hline \multicolumn{6}{|l|}{ Rural } \\
\hline Urban & $1.05(0.94-1.16)$ & $0.98(0.75-1.30)$ & $1.07(0.94-1.22)$ & $0.99(0.79-1.23)$ & $1.04(0.86-1.27)$ \\
\hline Intermediate & $0.83(0.75-0.92)$ & $0.52(0.41-0.67)$ & $1.05(0.92-1.19)$ & $0.80(0.65-0.99)$ & $0.78(0.64-0.94)$ \\
\hline \multicolumn{6}{|l|}{ Age } \\
\hline$<81$ years old & $1.01(1.00-1.01)$ & $1.04(1.03-1.05)$ & $1.01(1.01-1.02)$ & $1.01(1.00-1.03)$ & $0.99(0.98-1.01)$ \\
\hline$\geq 81$ years old & $0.91(0.90-0.92)$ & $0.89(0.87-0.92)$ & $0.89(0.88-0.90)$ & $0.97(0.95-1.00)$ & $0.92(0.90-0.94)$ \\
\hline Sex & $1.01(0.94-1.08)$ & $0.77(0.66-0.92)$ & $1.04(0.96-1.13)$ & $0.87(0.75-1.00)$ & $1.07(0.94-1.22)$ \\
\hline Charlson Comorbidity Index & $0.96(0.94-0.98)$ & $0.98(0.93-1.03)$ & $0.95(0.93-0.97)$ & $0.95(0.92-0.99)$ & $1.02(0.99-1.06)$ \\
\hline Total dependency ratio & $1.01(1.00-1.01)$ & $1.00(0.99-1.00)$ & $1.01(1.01-1.02)$ & $1.00(0.99-1.01)$ & $1.00(0.99-1.01)$ \\
\hline Constant & 2.21 & 5.46 & 2.38 & 12.75 & 47.34 \\
\hline
\end{tabular}

Results obtained from binary logistic regressions for the association living typology (rural versus intermediate versus urban) and basic examination, MMSE scores, Clock Test, Blood Analysis, and CT and/or MRI stratified for type of care (primary versus specialist). Results are adjusted for age, sex, Charlson Comorbidity Index, and total dependency ratio.

Furthermore, results of the stratified analyses showed differences in the diagnostic work-up processes between primary and specialist care, indicating that in primary care, patients living in rural areas receive more basic examination, MMSE testing, Clock tests, blood analyses, and imaging, whereas in specialist care these differences disappear. This is probably due to urban areas having higher availability of specialist units, with more patients directly referred to specialist care from primary care without a prior basic examination, which is then performed in the specialist unit. Also, the affiliation of primary care units to SveDem in urban areas are lower than in rural areas.

The present study also showed clear sex differences in nearly all investigated diagnostic work-up processes (except MMSE performance in rural areas), with men receiving more diagnostic work-up than women. It should be noted that women in our sample were older and showed a lower MMSE score compared to men over all three living typologies, as also supported by a review of Li and Singh [19]. These differences were also seen in an earlier report from SveDem where men were diagnosed with more technical investigations, but the gender differences disappeared when adjusted for age [20]. Even though clear sex differences have been reported in the incidence and prevalence of dementia [21], the reasons for these differences are still unclear [19]. Two contributory factors may be that age and lower MMSE do lead to overall fewer investigations performed in the diagnostic work-up [20]. Also living conditions may play a role. Another recent paper of ours showed that persons who live alone (the majority is women) get diagnosed later (i.e., have a lower MMSE at the time of diagnosis [22]).

The present study had several limitations. As already mentioned above, even though the coverage of SveDem is increasing, it is still not complete and was estimated at 36\% in 2012 [9]. Therefore, if indeed patients from rural areas have worse access to health care that could also lead to lower inclusion rates in SveDem and consequently to a sampling bias [2].

Furthermore, there are several different possible definitions of rural, intermediate, and urban living areas used in studies on living typologies. Therefore, data from a single study in one geographical location cannot be directly compared with those of another 
study from another country because of methodological differences and different definitions being used between the studies [1]. The variable used for the definition of living typology in the present study was defined according to the newest Swedish definition of rural, intermediate, and urban living typology and therefore allows important conclusions regarding the diagnostic work-up within Sweden. However, the general results regarding differences in urban and rural areas are still of high importance for health care providers outside of Sweden, as geographical variation is a factor of concern seen in several international studies [1].

Another limitation is the absence of information on other possible factors influencing the diagnostic work-up processes in rural, intermediate, and urban areas (e.g., neighborhood effects and segregation, immigration, social factors, ethnicity, and gender). Also, recent research shows that there are some environmental factors (e.g., total amount of green space [23], environmental pollution [24], and noise exposure [25]) that could influence the development and progression of dementia in rural, intermediate, and urban living areas, which could serve as possible controlling variables. Therefore, further studies should take these variables into account when examining dementia and living typology.

The large sample size is a particular strength of the present study. It includes individuals from throughout the whole country and therefore reflects epidemiological reality.

This is the first study that examines differences in diagnostic work-up processes in patients with dementia in rural, intermediate, and urban areas in Sweden. As already mentioned, the variable used for the definition of living typology was defined according to the newest definition of rural, intermediate, and urban living typology. Thus, it is ensured that the classification is relevant for the Swedish setting.

The identification of differences in diagnostic work-up processes in rural, intermediate, and urban areas is of considerable interest to assess the quality of diagnostic work-up for patients with dementia in Sweden and possibly other countries. The results of the present study should be taken into consideration in future healthcare decisions to ensure equality of health care across rural, intermediate, and urban areas to assess inequalities regarding diagnostic work-up processes in the different living typologies (e.g., by sensitizing urban practioners to use further diagnostic processes, or by starting information campaigns on sex differences in diagnostic treatment). The future aim should be to avoid geographical and sex differences in diagnostic work-up processes.

\section{ACKNOWLEDGMENTS}

SveDem is supported financially by the Swedish Associations of Local Authorities and Regions. This study was supported by the Swedish Research Council for Health, Working Life and Welfare (FORTE grant \# 2017-01646), the Swedish Research Council (grant \# 2016-02317), Svenska Sällskapet för Medicinsk Forskning/The Swedish Society for Medical Research, the Swedish Order of St John and grants from the Regional Agreement on Medical Training and Clinical Research (ALF) between Stockholm County Council and the Karolinska Institutet.

Authors' disclosures available online (https:// www.j-alz.com/manuscript-disclosures/19-0017r1).

\section{SUPPLEMENTARY MATERIAL}

The supplementary material is available in the electronic version of this article: http://dx.doi.org/ 10.3233/JAD-190017.

\section{REFERENCES}

[1] Russ TC, Batty GD, Hearnshaw GF, Fenton C, Starr JM (2012) Geographical variation in dementia: Systematic review with meta-analysis. Int J Epidemiol 41, 1012-1032.

[2] Ehrlich K, Boström AM, Mazaheri M, Heikkilä K, Emami A (2015) Family caregivers' assessments of caring for a relative with dementia: A comparison of urban and rural areas. Int J Older People Nurs 10, 27-37.

[3] Liu Z, Albanese E, Li S, Huang Y, Ferri CP, Yan F, Sousa R, Dang W, Prince M (2009) Chronic disease prevalence and care among the elderly in urban and rural Beijing, Chinaa 10/66 Dementia Research Group cross-sectional survey. BMC Public Health 9, 394.

[4] Nordberg G (2007) Formal and informal care in an urban and a rural elderly population: Who? When? What?, Institutionen för neurobiologi, vårdvetenskap och samhälle/Department of Neurobiology, Care Sciences and Society.

[5] Roheger M, Zupanic E, Kåreholt I, Religa D, Kalbe E, Eriksdotter M, Garcia-Ptacek S (2018) Mortality and nursing home placement of dementia patients in rural and urban areas: A cohort study from the Swedish Dementia Registry. Scand J Caring Sci 32, 1308-1313.

[6] Swedish National Board of Health and Welfare (2010) National Guidelines for the Care of Cases of Dementia. Swedish National Board of Health and Welfare, Sweden.

[7] Folstein MF, Folstein SE, McHugh PR (1975) "Mini-mental state": A practical method for grading the cognitive state of patients for the clinician. J Psychiatr Res 12, 189-198.

[8] Rouleau I, Salmon DP, Butters N (1996) Longitudinal analysis of clock drawing in Alzheimer's disease patients. Brain Cogn 31, 17-34. 
[9] Religa D, Fereshtehnejad S-M, Cermakova P, Edlund A-K, Garcia-Ptacek S, Granqvist N, Hallbäck A, Kåwe K, Farahmand B, Kilander L (2015) SveDem, the Swedish Dementia Registry-a tool for improving the quality of diagnostics, treatment and care of dementia patients in clinical practice. PloS One 10, e0116538.

[10] Garcia-Ptacek S, Farahmand B, Kåreholt I, Religa D, Cuadrado ML, Eriksdotter M (2014) Mortality risk after dementia diagnosis by dementia type and underlying factors: A cohort of 15,209 patients based on the Swedish Dementia Registry. J Alzheimers Dis 41, 467-477.

[11] Wettermark B, Hammar N, MichaelFored C, Leimanis A, Olausson PO, Bergman U, Persson I, Sundström A, Westerholm B, Rosén M (2007) The new Swedish Prescribed Drug Register-opportunities for pharmacoepidemiological research and experience from the first six months. Pharmacoepidemiol Drug Safety 16, 726-735.

[12] Ludvigsson JF, Andersson E, Ekbom A, Feychting M, Kim J-L, Reuterwall C, Heurgren M, Olausson PO (2011) External review and validation of the Swedish national inpatient register. BMC Public Health 11, 450.

[13] Omarbetning av Sveriges Kommuner och Landstings kommungruppsindelning, Kommungruppsindelning, http://web butik.skl.se/sv/artiklar/kommungruppsindelning-2017.html \#sthash.37iAvhQF.dpuf, Accessed 10 June.

[14] SCB, SCB Statistics, http://www.statistikdatabasen.scb.se/ pxweb/sv/ssd/START_AM_AM9906__AM9906D/Region IndR2/?rxid=d7bdac60-e800-4923-81dd-8200abedca52, Accessed 10 June.

[15] Charlson ME, Pompei P, Ales KL, MacKenzie CR (1987) A new method of classifying prognostic comorbidity in longitudinal studies: Development and validation. J Chronic Dis 40, 373-383.

[16] Corp I (2013) IBM SPSS statistics for windows, version 22.0. Armonk, NY: IBM Corp.

[17] Garcia-Ptacek S, Modéer IN, Kåreholt I, Fereshtehnejad S-M, Farahmand B, Religa D, Eriksdotter M (2016) Differences in diagnostic process, treatment and social Support for Alzheimer's dementia between primary and specialist care: Resultss from the Swedish Dementia Registry. Age Ageing 46, 314-319.
[18] SveDem (2017) SveDem yearly report.

[19] Li R, Singh M (2014) Sex differences in cognitive impairment and Alzheimer's disease. Front Neuroendocrinol 35, 385-403.

[20] Religa D, Spångberg K, Wimo A, Edlund A-K, Winblad B, Eriksdotter-Jönhagen M (2012) Dementia diagnosis differs in men and women and depends on age and dementia severity: Data from SveDem, the Swedish Dementia Quality Registry. Dement Geriatr Cogn Disord 33, 90-95.

[21] De Deyn P, Goeman J, Vervaet A, Dourcy-Belle-Rose B, Van Dam D, Geerts E (2011) Prevalence and incidence of dementia among 75-80-year-old community-dwelling elderly in different districts of Antwerp, Belgium: The Antwerp Cognition (ANCOG) Study. Clin Neurol Neurosurg 113, 736-745.

[22] Cermakova P, Nelson M, Secnik J, Garcia-Ptacek S, Johnell K, Fastbom J, Kilander L, Winblad B, Eriksdotter M, Religa D (2017) Living alone with Alzheimer's disease: data from SveDem, the Swedish Dementia Registry. J Alzheimers Dis 58, 1265-1272.

[23] Cherrie MP, Shortt NK, Mitchell RJ, Taylor AM, Redmond P, Thompson CW, Starr JM, Deary IJ, Pearce JR (2018) Green space and cognitive ageing: A retrospective life course analysis in the Lothian Birth Cohort 1936. Social Sci Med 196, 56-65.

[24] Chin-Chan M, Navarro-Yepes J, Quintanilla-Vega B (2015) Environmental pollutants as risk factors for neurodegenerative disorders: Alzheimer and Parkinson diseases. Front Cell Neurosci 9, 124.

[25] Münzel T, Gori T, Babisch W, Basner M (2014) Cardiovascular effects of environmental noise exposure. Eur Heart $J$ 35, 829-836. 